\title{
APPROXIMATION OF DISCONTINUOUS FUNCTIONS OF TWO VARIABLES BY DISCONTINUOUS INTERLINATION SPLINES USING TRIANGULAR ELEMENTS
}

\author{
V. Mezhuyev ${ }^{1 *}$, O. M. Lytvyn², I. Pershyna², O. Nechuiviter ${ }^{2}$ \\ ${ }^{1}$ FH JOANNEUM University of Applied Sciences, Kapfenberg, Austria \\ e-mail: vitaliy.mezhuyev@ fh-joanneum.at \\ ${ }^{2}$ Department of Information Computer Technologies and Mathematics, Ukrainian Engineering \\ and Pedagogical Academy, Kharkiv, Ukraine \\ e-mail: yuliapershina78@gmail.com \\ *corresponding author
}

\begin{abstract}
The paper develops a method for approximation of the discontinuous functions of two variables by discontinuous interlination splines using arbitrary triangular elements. Experimental data are one-sided traces of a function given along a system of lines (such data are commonly used in remote methods, in particular in tomography). The paper also proposes a method for approximating the discontinuous functions of two variables taking into account triangular elements having one curved side. The proposed methods improve approximation of the discontinuous functions, allowing an application to complex domains of definition and avoiding the Gibbs phenomenon.
\end{abstract}

Keywords: Discontinuous function, Discontinuous interlination, Gibbs phenomenon, Computer tomography, Triangular elements

\section{Introduction}

The methods of approximation of smooth functions by continuous methods with sufficient completeness were considered in works of many researchers [1, 2]. However, as a rule, existing methods consider only exceptional cases or the results of extreme idealization. At the same time, real objects can be adequately described only by the functions with discontinuities, kinks, or other violations of smoothness. Examples of such nonsmooth functions are the profiles of shock waves generated by acoustic emitters and the shape of dunes in the desert, which have a characteristic outline, etc. Discontinuous objects are common idealisations in the problems, which are commonly solved with remote methods. E.g., discovery of cracks in industrial products using non-destructive testing is an important task in flaw detection, aimed at determining deviations of an internal structure of a product. In the problems of geophysics, detection of the boundaries of the blocks with different physical properties is the first step of the study of the physical characteristics of the internal structure of the Earth. At the study of an internal structure of a body in computed tomography, the heterogeneity (different densities of the various parts of the body) should be also taken into account. 
In general, remote methods become one of the promising areas of the environmental studies. They serve as an important source of information about various phenomena occurring in the geographic shell of the Earth and are an effective tool for monitoring the state of the environment and solving environmental problems. It is obvious that further progress in the development of natural sciences is linked with the use of remote research methods.

This work belongs to the series of works of the authors aimed to study and improve mathematical models in computed tomography [3, 4]. Currently, tomography has developed many computational methods, algorithms and software tools aimed at reconstruction the internal properties of an object. They perform well when restoring objects with smooth properties, but produce unsatisfactory results for objects having discontinuous characteristics. Therefore, there is a need to improve the mathematical methods for approximating discontinuous functions, allowing for increase an accuracy of the models of reconstructed objects.

The mathematical foundations of tomography were laid in the works of the German scientist J. Radon [5], who developed the theory of the transformation of functions of many variables (Radon transformation). According to these transformations, the function of many variables can be characterized not only by its values at the points of multidimensional space but also by the integrals from this function taken over an infinite set of lines or planes.

The approximation of discontinuous functions by trigonometric Fourier sums causes so called the Gibbs phenomenon. Various filters have been developed to overcome this phenomenon $[6,7]$. Some authors $[8,9]$ proposed the methods for reconstruction of discontinuous lines using wavelets. There are also works that propose the use of direct and inverse Radon transform for reconstruction of discontinuities in computed tomography. Faridani, Finch, Ritman, Smith [10], Louis [11], and Maass further developed this methodology and tools for algorithmic reconstruction of discontinuities in computed tomography. Ramachandran, Lakshminarayanan, and Ramm proposed approaches that allow restoring not only a set of discontinuities, but also the jump values by Radon transform [12]. Further development of this approach can be found in [13, 14]. These works are based on the direct and inverse Radon transform (direct Radon transform is an integral transformation and the derived functions, having a higher smoothness than the converted function).

At the same time, existing filters and methods cannot completely overcome the Gibbs phenomenon. The Italian scientist Rossini M. [15] developed the methods for reconstruction of discontinuous lines using wavelets. These recovery methods use polyharmonic wavelets that have an infinite carrier. It leads to a smoothing of the study signal but require additional analysis of the results.

The methods for solving boundary-value problems with discontinuous solutions have been contributed by Sergienko I.V., Deineka V.S., Skopetsky V.V., Lytvyn O.M. A.L. Ageeva and T.V. Antonov $[16,17]$ proposed a method for determining the number of breakpoints and their positions based on the use of the Gibbs phenomenon. However, the method requires additional information: the smallest and largest values of the jumps of an approximate function. It is also assumed that the intervals, in which the Gibbs phenomena arises, do not intersect; i.e. it is impossible to separate the breakpoints that are close to each other.

The series of works by the authors [18-20] were devoted to solving the flat problem of Radon computed tomography using heterogeneity of the internal structure of a two-dimensional body. Here, it was advised to use interlination operators, since these operators restore (possibly approximated) functions by their known traces on a given system of lines. Researchers provide a method to construct operators, whose integrals from the lines (linear integrals) will be equal to integrals from the most renewable function. Note that interlination is a mathematical apparatus, 
naturally related to the problem of restoring the characteristics of objects according to their known projections. This paper gives an elaboration of the methods described in [18-20].

The study [18] proposed the method for the restoration of the discontinuous functions of one variable and the algorithm for detecting $\varepsilon$-discontinuity points. In $[19,20]$, an algorithm for detecting the lines of $\varepsilon$-discontinuity was developed, allowing a separation of the functions of two variables with the help of discontinuous approximation splines and rectangular elements. In [21], a method for solving the 2D problem of computed tomography using the inhomogeneity of an internal structure of a body was proposed. To solve it, the paper [21] constructed the discontinuous interlination operator based on known one-sided traces of a function along a system of given mutually perpendicular lines. This paper constructs the discontinuous interlineation operator from the known traces of the function of two variables on a system of arbitrary lines that do not intersect at one point.

\section{Formulation of the Problem}

Let the function $f(x, y)$ be a discontinuous function of two variables in the domain $D$. We will assume that the domain $D$ is divided into arbitrary triangles. The sides of the triangles do not intersect. The function $f(x, y)$ has the first-order discontinuities at the boundaries between these triangular elements (but not necessarily at all). The experimental data are unilateral traces of a function along a system of the given lines. Based on these data, let us construct an analytic formula of an unknown function (i.e. the discontinuous interlination operator).

\section{Development of the method and formula}

Let us consider a triangular element $\mathrm{T}_{i j}, i=\overline{1, n}, j=\overline{1, m}$ (Fig 1), formed by straight lines

$$
\begin{aligned}
& \omega 1_{i}(x, y)=0, \quad \omega 2_{j}(x, y)=0, \quad \omega 3_{i j}(x, y)=0, \quad \omega 1_{i}:=x-x_{i}, \omega 2_{j}:=y-y_{j}, \\
& \omega 3_{i j}:=\frac{x-x_{i}}{x_{i+1}-x_{i}}-\frac{y-y_{j+1}}{y_{j}-y_{j+1}}, i=\overline{1, n}, j=\overline{1, m}
\end{aligned}
$$

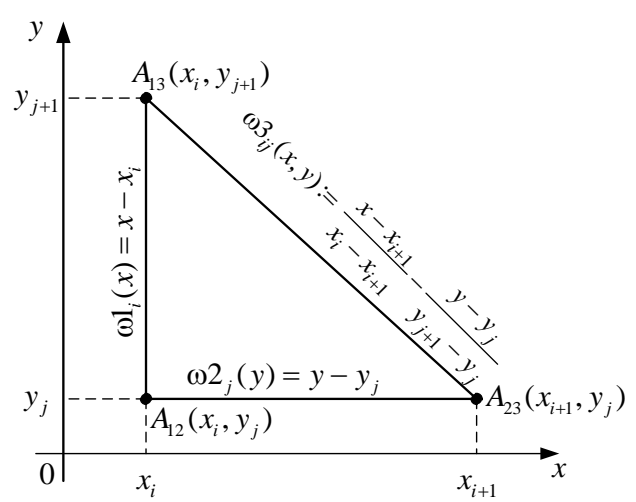

Fig. 1. View of a triangular element $T_{i j}$ 
Let us consider as given:

Function traces $f(x, y)$ on the straight line $x=x_{i}$ (on the right and on the left of the line respectively):

$$
\begin{aligned}
& \varphi p_{i}(y)=\lim _{x \rightarrow x_{i}+0} f(x, y), \varphi m_{i}(y)=\lim _{x \rightarrow x_{i}-0} f(x, y) ; \\
& \varphi p p_{i j}=\varphi p_{i}\left(y_{j}\right)=\lim _{\substack{x \rightarrow x_{i}+0 \\
y \rightarrow y_{j}+0}} f(x, y), \varphi m p_{i j}=\varphi m_{i}\left(y_{j}\right)=\lim _{\substack{x \rightarrow x_{i}-0 \\
y \rightarrow y_{j}+0}} f(x, y)
\end{aligned}
$$

Function traces on the straight line $y=y_{j}$ (on the right and on the left of the line respectively). Formulas are developed similarly to the traces along the line $x=x_{i}$.

Function traces on the straight line $y=\frac{\left(y_{j}-y_{j+1}\right)\left(x-x_{i}\right)}{x_{i+1}-x_{i}}+y_{j+1}$ (on the right and on the left of the line respectively):

$$
\begin{gathered}
\eta m_{i j}(x)=f\left(x, \frac{\left(y_{j}-y_{j+1}\right)\left(x-x_{i}\right)}{x_{i+1}-x_{i}}+y_{j+1}-0\right), \\
\eta p_{i j}(x)=f\left(x, \frac{\left(y_{j}-y_{j+1}\right)\left(x-x_{i}\right)}{x_{i+1}-x_{i}}+y_{j+1}+0\right), \eta p m_{i j}=\eta m_{i j}\left(x_{i}\right)=f\left(x_{i}+0, y_{j+1}-0\right), \\
\eta p p_{i j}=\eta p_{i j}\left(x_{i}\right)=f\left(x_{i}+0, y_{j+1}+0\right)
\end{gathered}
$$

or

$$
\begin{gathered}
\eta m_{i j}(y)=f\left(\frac{\left(y-y_{j}\right)\left(x_{i}-x_{i+1}\right)}{y_{j+1}-y_{j}}+x_{i+1}+0, y\right), \eta p_{i j}(y)=f\left(\frac{\left(y-y_{j}\right)\left(x_{i}-x_{i+1}\right)}{y_{j+1}-y_{j}}+x_{i+1}-0, y\right), \\
\eta p m_{i j}=\eta m_{i j}\left(y_{j}\right)=f\left(x_{i+1}+0, y_{j}-0\right), \eta \eta p_{i j}=\eta p_{i j}\left(y_{j}\right)=f\left(x_{i+1}-0, y_{j}+0\right) .
\end{gathered}
$$

Theorem 1. Let the traces of the function $f(x, y)$ satisfy the conditions $\psi p_{j}\left(x_{i}\right)=\varphi p_{i}\left(y_{j}\right), \quad \eta m_{i j}\left(x_{i}\right)=\varphi p_{i}\left(y_{j+1}\right), \eta m_{i j}\left(x_{i+1}\right)=\psi p_{j}\left(x_{i+1}\right)$. That is, the tracks at the intersection points of the lines coincide. Then the operator

$$
\begin{gathered}
O f(x, y)=\frac{\omega 3_{i j}(x, y)}{\omega 3_{i j}\left(A_{12}\right)}\left(\psi p_{j}(x)+\varphi p_{i}(y)-\varphi p_{i}\left(y_{j}\right)\right)+\frac{\omega 2_{j}(y)}{\omega 2_{j}\left(A_{13}\right)}\left(\eta m_{i j}(x)-\right. \\
\left.-\varphi p_{i}\left(y_{j}+\frac{\left(x-x_{i+1}\right)\left(y_{j+1}-y_{j}\right)}{\left(x_{i}-x_{i+1}\right)}\right)+\varphi p_{i}(y)\right)+\frac{\omega 1_{i}(x)}{\omega 1_{i}\left(A_{23}\right)} \times \\
\times\left(\eta m_{i j}\left(x_{i+1}+\frac{\left(y-y_{j}\right)\left(x_{i}-x_{i+1}\right)}{\left(y_{j+1}-y_{j}\right)}\right)-\psi p_{j}\left(x_{i+1}+\frac{\left(y-y_{j}\right)\left(x_{i}-x_{i+1}\right)}{\left(y_{j+1}-y_{j}\right)}\right)+\psi p_{j}(x)\right)
\end{gathered}
$$

is the operator of interlination of a function $f(x, y)$ on $\partial \mathrm{T}_{i}:\left.O f(x, y)\right|_{\partial \mathrm{T}_{i}}=\left.f(x, y)\right|_{\partial \mathrm{T}_{i}}$. 
Theorem 2. If $f(x, y)$ is a continuous function (together with its partial derivatives up to the second order inclusively) inside a triangular element $\mathrm{T}_{i j}$, then for the residual $R f(x, y)=(I-O) f$ equals

$$
\begin{aligned}
& R f(x, y)=\frac{\omega 3_{i j}(x, y)}{\omega 3_{i j}\left(A_{12}\right)} \\
& \int_{x_{i}}^{x} \int_{y_{j}}^{y} f^{(1,1)}(u, v) d u d v+\frac{\omega 2_{j}(y)}{\omega 2_{j}\left(A_{13}\right)} \int_{x_{i}}^{x} \int_{y_{j}+\frac{\left(x-x_{i+1}\right)\left(y_{j+1}-y_{j}\right)}{x_{i}-x_{i+1}}}^{y} f^{(1,1)}(u, v) d u d v \\
& +\frac{\omega 1_{i}(x)}{\omega 1_{i}\left(A_{23}\right)} \int_{x_{i+1}+\frac{\left(y-y_{j}\right)\left(x_{i}-x_{i+1}\right)}{\left(y_{j+1}-y_{j}\right)}}^{x} \int_{y_{j}}^{y} f^{(1,1)}(u, v) d u d v,(x, y) \in \mathrm{T}_{i j} .
\end{aligned}
$$

In the case, when the domain is triangulated, the residual of interlination in each triangle does not equal the product of the residuals of one-dimensional interpolation, in contrast to the case of dividing the domain into rectangular elements.

Let us estimate the error of the formula, which general form was obtained in theorem 2.

Theorem 3. Let $f(x, y) \in L_{\infty}^{1,1}\left(\mathrm{~T}_{i j}\right), \forall(x, y) \in \mathrm{T}_{i j}$, then there the following error estimates $\forall(x, y) \in \mathrm{T}_{i j}$, :

$$
|R f(x, y)| \leq\left\|f^{(1,1)}(x, y)\right\|_{L_{\infty}\left(\mathrm{T}_{i j}\right)}\left|\left(x-x_{i}\right)\left(y-y_{j}\right)\left(\frac{x-x_{i+1}}{x_{i}-x_{i+1}}-\frac{y-y_{j}}{y_{j+1}-y_{j}}\right)\right|,
$$

where $L_{\infty}^{1,1}\left(\mathrm{~T}_{i j}\right)=\lim _{p \rightarrow \infty} L_{p}\left(\mathrm{~T}_{i j}\right)=\sup v \operatorname{rai}|f(x, y)|-$ significant upper bound of the function $|f(x, y)|$ on $\mathrm{T}_{i j}$, that is, the smallest of the numbers $K \geq 0$ for which the inequality $|f(x, y)|>K$ holds on the set of measure zero.

Theorem 4. If the conditions of Theorem 2 and 3 are satisfied, then the function

$$
f(x, y)=\left(x-x_{i}\right)\left(y-y_{j}\right)\left(\frac{x-x_{i+1}}{x_{i}-x_{i+1}}-\frac{y-y_{j}}{y_{j+1}-y_{j}}\right)
$$

turns the inequality (1) into an equality.

Example 1. Let $\mathrm{T}=\{x, y>0: 1-x-y>0\}$ is a domain of the definition of the function $f(x, y)$. The function is continuously differentiable inside a given triangle $\mathrm{T}$. Then, by theorem 1 , the operator of polynomial interlination will have the form

$$
\begin{gathered}
O f(x, y)=(1-x-y)(\psi p p(x)+\varphi p(y)-\varphi p(0))+y(\eta m(x)-\varphi p(1-x)+\varphi p(y))+ \\
+x(\eta m(1-y)-\psi p(1-y)+\psi p(x)) .
\end{gathered}
$$

The error, estimated by Theorem 3 , has the form

$$
\left|R_{0} f(x, y)\right| \leq|x y(1-x-y)|, \forall(x, y) \in \mathrm{T}, \forall f(x, y) \in L_{\infty}^{1,1}(\mathrm{~T}) .
$$


The value $|x y(1-x-y)|$ is always positive, so we can estimate the above value, by finding the largest value of the function $f(x, y)$ :

$$
\left\{\begin{array} { l } 
{ f _ { x } ^ { \prime } = 0 } \\
{ f _ { y } ^ { \prime } = 0 }
\end{array} \Rightarrow \left\{\begin{array} { l } 
{ y ( 1 - x - y ) - x y = 0 } \\
{ x ( 1 - x - y ) - x y = 0 }
\end{array} \Rightarrow \left\{\begin{array}{l}
y-2 x y-y^{2}=0, \\
x-2 x y-x^{2}=0 .
\end{array}\right.\right.\right.
$$

Having solved this system, we obtain four stationary points $(0,0),(1 / 3 ; 1 / 3),(0,1),(1,0)$. After substituting points in the function $f(x, y)=x y|1-x-y|$, we find the largest value of this function, equal to $1 / 27$, at the point $(1 / 3 ; 1 / 3)$. Therefore $\left|R_{0} f(x, y)\right| \leq|x y(1-x-y)| \leq 1 / 27$.

Example 2. Let the function $f(x, y)$ be defined in the region $\mathrm{T}=\mathrm{T}_{1} \cup \mathrm{T}_{2} \cup \mathrm{T}_{3} \cup \mathrm{T}_{4}$ shown in Fig. 2:

$$
\begin{aligned}
& \mathrm{T}_{1}=\{x, y>0,1-x-y>0\}, \mathrm{T}_{2}=\{x<0, y>0,1+x-y>0\}, \\
& \mathrm{T}_{3}=\{x, y<0,1+x+y>0\}, \mathrm{T}_{4}=\{x>0, y<0,1-x+y>0\}
\end{aligned}
$$

Let the function $f(x, y)$ have discontinuities of the first kind on the lines of triangulation and let the following traces be given on these lines:

$$
\begin{gathered}
\varphi p(y)=f(+0, y)=y, \quad \varphi m(y)=f(-0, y)=-y, \\
\psi p(x)=f(x,+0)=2 x, \quad \psi m(x)=f(x,-0)=x, \\
\eta m_{1}(x)=f(x, 1-x-0)=1+x, \\
\eta m_{2}(x)=f(x, 1+x-0)=-1+x, \\
\eta m_{3}(x)=f(x,-1-x-0)=1+2 x, \\
\eta m_{4}(x)=f(x,-1+x-0)=-1+2 x .
\end{gathered}
$$

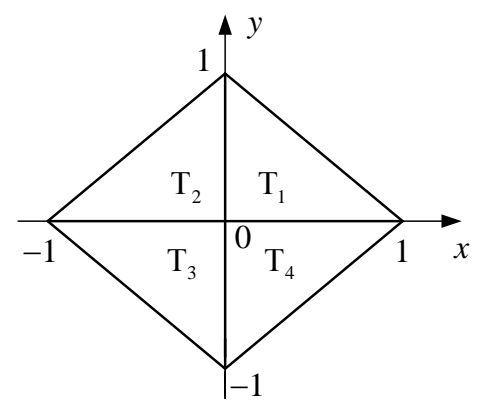

Fig. 2. The area of the approximated function $f(x, y)$

These traces satisfy the conditions of Theorem 1 in each of the four triangles. The discontinuous spline-interlinant will be constructed in the form, represented by the formula (1) 


$$
S(x, y)= \begin{cases}O_{1} f(x, y), & (x, y) \in \mathrm{T}_{1} \\ O_{2} f(x, y), & (x, y) \in \mathrm{T}_{2} \\ O_{3} f(x, y), & (x, y) \in \mathrm{T}_{3} \\ O_{4} f(x, y), & (x, y) \in \mathrm{T}_{4}\end{cases}
$$

The operators $O_{1}, O_{2}, O_{3}, O_{4}$ are calculated by the formula (1). Let us consider in detail the construction of the operator $O_{1}(x, y)$ on a triangular element $\mathrm{T}_{1}=\{x, y>0,1-x-y>0\}$. According to the formula (1), the operator $O_{1}(x, y)$ has the form

$$
\begin{gathered}
O_{1} f(x, y)=(1-x-y)(\psi p(x)+\varphi p(y)-\varphi p(0))+y\left(\eta m_{1}(x)-\varphi p(1-x)+\varphi p(y)\right)+ \\
x\left(\eta m_{1}(1-y)-\psi p(1-y)+\psi p(x)\right)
\end{gathered}
$$

where $\psi p(x)$ is a one-way trace of the function to be restored, above the line $y=0$ (by condition $\psi p(x)=2 x) ; \varphi p(y)$ is a one-way function trace right of the line $x=0$ ( by condition $\varphi p(y)=y) ; \eta m_{1}(x)$ is a one-way function trace to the left of the line $y=1+x$ (by condition $\left.\eta m_{1}(x)=1+x\right)$.

By analogy are constructed the operators $\mathrm{O}_{2}, \mathrm{O}_{3}, \mathrm{O}_{4}$

$$
\begin{gathered}
O_{2} f(x, y)=(1+x-y)(\psi p(x)+\varphi m(y)-\varphi m(0))+y\left(\eta m_{2}(x)-\varphi m(1+x)+\varphi m(y)\right)- \\
-x\left(\eta m_{2}(-1+y)-\psi p(-1+y)+\psi p(x)\right), \\
O_{3} f(x, y)=(1+x+y)(\psi m(x)+\varphi m(y)-\varphi m(0))-y\left(\eta m_{3}(x)-\varphi m(-1-x)+\varphi m(y)\right)- \\
-x\left(\eta m_{3}(-1-y)-\psi m(-1-y)+\psi m(x)\right), \\
O_{4} f(x, y)=(1-x+y)(\psi m(x)+\varphi p(y)-\varphi p(0))-y\left(\eta m_{4}(x)-\varphi p(-1+x)+\varphi p(y)\right)+ \\
+x\left(\eta m_{4}(1+y)-\psi m(1+y)+\psi m(x)\right) .
\end{gathered}
$$

We substitute the value of traces of the function $f(x, y)$ on the corresponding lines into the resulting discontinuous spline. As a result, we get

$$
\begin{gathered}
S(x, y)=\left\{\begin{array}{l}
(1-x-y)(2 x+y)+y(1+x-1+x+y)+x(1+1-y-2+2 y+2 x),(x, y) \in \mathrm{T}_{1}, \\
(1+x-y)(2 x-y)+y(-1+x+1+x-y)-x(-1-1+y+2 x-2 y),(x, y) \in \mathrm{T}_{2}, \\
(1+x+y)(x-y)-y(1+2 x-1-x-y)-x(1-2-2 y+1+y+x),(x, y) \in \mathrm{T}_{3}, \\
(1-x+y)(x+y)-y(-1+2 x+1-x)+x(-1+2+2 y-1-y+x),(x, y) \in \mathrm{T}_{4},
\end{array}\right. \\
\begin{cases}2 x+y, & (x, y) \in \mathrm{T}_{1}, \\
2 x-y, & (x, y) \in \mathrm{T}_{2}, \\
x-y, & (x, y) \in \mathrm{T}_{3}, \\
x+y, & (x, y) \in \mathrm{T}_{4} .\end{cases}
\end{gathered}
$$

It can be seen that the function $S(x, y)$ on the border between the elements $\mathrm{T}_{1}$ and $\mathrm{T}_{4}$ will have the following traces:

$$
S(+0, y)=(1-y)(\psi p(0)+\varphi p(y)-\varphi p(0))+y\left(\eta m_{1}(0)-\varphi p(1)+\varphi p(y)\right), \quad(x, y) \in \mathrm{T}_{1} ;
$$




$$
S(-0, y)=(1-y)(\psi p(0)+\varphi m(y)-\varphi m(0))++y\left(\eta m_{2}(0)-\varphi m(1)+\varphi m(y)\right),(x, y) \in \mathrm{T}_{2} .
$$

That is, if $\varphi p(0) \neq \varphi m(0), \varphi p(1) \neq \varphi m(1)$, then the function $f(x, y)$ will be discontinuous on the line $x=0$.

\section{Approximation of the Discontinuous Function of Two Variables by a Discontinuous Interlination Spline and Triangular Elements with One Curved Side}

Let a discontinuous function of two variables $f(x, y)$ be defined in the domain $D$. We assume that domain $D$ is divided by lines $x_{0}=0<x_{1}<x_{2}<\ldots<x_{m}=1, \quad y_{0}=0<y_{1}<y_{2}<\ldots$ $. .<y_{n}=1$, into rectangular elements, and each rectangle is divided into two right triangles with curvilinear hypotenuse. Triangles do not fit into each other, and the sides of the triangles do not intersect. The function $f(x, y)$ has discontinuities of the first kind at the boundaries between these right triangles (not necessarily between all). Let us construct an operator of discontinuous piecewise polynomial interlination, such that in each triangle it is an operator of polynomial interlination of a function $f(x, y)$.

Consider a triangular element $\mathrm{T}_{i j}, i=\overline{1, n}, j=\overline{1, m}$ (Fig. 3), in which the catheti are defined by the equations: $A B: x=x_{i}, A C: y=y_{j}$, and the hypotenuse $B C$ is curved and can be defined by an equation $h(x)+g(y)=1$, that is $y=g^{-1}(1-h(x))$ or $x=h^{-1}(1-g(y))$. In addition, the following relationships are fulfilled $g\left(y_{j}\right)=0, h\left(x_{i}\right)=0$.

Let a function $f(x, y)$ is given on this triangle, which may have discontinuities of the first kind on the lines of a given triangular element.

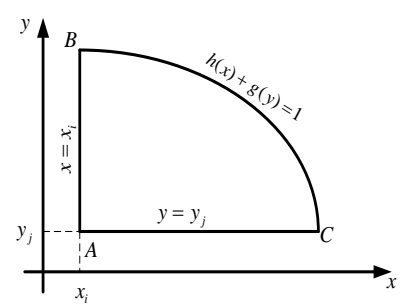

Fig. 3. Example of a triangular element with curvilinear hypotenuse and with a right angle

Let us consider the given: 1$)$ traces of the function $f(x, y)$ on the line $x=x_{i}$ (to the right and to the left of the line); 2) traces of the function $f(x, y)$ on the line $y=y_{j}$ (to the right and to the left of the line); 3 ) traces of a function $f(x, y)$ on a curved hypotenuse (below and above of the line):This formulas are developed similarly to the item III.

We introduce the following notation:

$$
\begin{array}{cc}
\mu m_{i}(x) & =f\left(x_{i}+0, g^{-1}(1-h(x))-0\right), \quad \mu p_{i}(x)=f\left(x_{i}+0, g^{-1}(1-h(x))+0\right) \\
\text { or } \mu p_{j}(y) & =f\left(h^{-1}(1-g(y))+0, y_{j}-0\right), \quad \mu m_{j}(y)=f\left(h^{-1}(1-g(y))-0, y_{j}+0\right) .
\end{array}
$$


It is easy to verify that the following relations hold:

$$
\begin{array}{ll}
\mu m_{i}\left(x_{i}\right)=\eta m_{i j}\left(x_{i}\right), & \mu p_{i}\left(x_{i}\right)=\eta p_{i j}\left(y_{j}\right), \\
\mu p_{j}\left(y_{j}\right)=\eta m_{i j}\left(y_{j}\right), & \mu m_{j}\left(y_{j}\right)=\eta p_{i j}\left(y_{j}\right) .
\end{array}
$$

Let us construct the operator of interlination.

Theorem 5. If traces of the function $f(x, y)$ satisfy the conditions

$$
\begin{gathered}
\psi p_{j}\left(x_{i}\right)=\varphi p_{i}\left(y_{j}\right), \quad \eta m_{i j}\left(x_{i}\right)=\varphi p_{i}\left(g^{-1}\left(1-h\left(x_{i}\right)\right)\right), \\
\eta m_{i j}\left(h^{-1}\left(1-g\left(y_{j}\right)\right)\right)=\psi p_{j}\left(h^{-1}\left(1-g\left(y_{j}\right)\right)\right),
\end{gathered}
$$

then the operator

$$
\begin{gathered}
L f(x, y)=L_{1} f(x, y)+L_{2}(x, y)-L_{12}(x, y) \\
L_{1} f(x, y)=h(x) \cdot \eta m_{i j}(y)+g(y) \cdot \eta m_{i j}(x), \\
L_{2} f(x, y)=\psi p_{j}(x)+\varphi p_{i}(y)-\varphi p_{i}\left(y_{j}\right), \\
L_{1} L_{2} f(x, y)=h(x)\left(\varphi p_{i}(y)+\mu p_{j}(y)-\varphi p_{i}\left(y_{j}\right)\right)+g(y)\left(\eta m_{i j}\left(x_{i}\right)+\mu m_{i}(x)-\psi p_{j}(x)\right),
\end{gathered}
$$

interlinated a function $f(x, y)$ on three sides of a triangle $\mathrm{T}_{i j}, i=\overline{1, n}, j=\overline{1, m}$, i.e.

$$
\begin{gathered}
\left.L f(x, y)\right|_{y=y_{j}}=\psi p_{j}(x),\left.L f(x, y)\right|_{x=x_{i}}=\varphi p_{i}(y), \\
L f(x, y)=f(x, y), \text { if } h(x)+g(y)=1 .
\end{gathered}
$$

Theorem 6. If $f(x, y) \in C^{(1,1)}\left(\mathrm{T}_{i j}\right)$, then for the residual $R f(x, y)=(I-L) f(x, y)$ equals

$$
\begin{gathered}
R f(x, y)=(1-h(x)-g(y)) \int_{0}^{x} \int_{0}^{y} f^{(1,1)}(u, v) d u d v+ \\
+f(x) \int_{x}^{h^{-1}(1-g(y))} \int_{0}^{y} f^{(1,1)}(u, v) d u d v+g(y) \int_{0}^{x} \int_{y}^{g^{-1}(1-h(x))} f^{(1,1)}(u, v) d u d v .
\end{gathered}
$$

Remark. For arbitrary functions $f(x, y)=u(x)+v(y)$, where $u(x), v(y)$ are arbitrary functions of one variable, the equality holds $L f(x, y)=f(x, y)$.

Comment. If $\varphi p_{i}(y)=\varphi m_{i}(y), \psi p_{j}(x)=\psi m_{j}(x), \eta p_{i j}(x)=\eta m_{i j}(x)$, then the constructed discontinuous spline of the form (3) is a continuous interlination spline at the boundaries of a triangular element $\mathrm{T}_{i j}$.

Example 3. Let the function $f(x, y)$ be defined in the domain $\mathrm{T}=\mathrm{T}_{1} \cup \mathrm{T}_{2} \cup \mathrm{T}_{3} \cup \mathrm{T}_{4}$ as shown in Fig. 4a. The catheti of these triangular elements are formed by straight lines $x=0, y=0$ , and the hypotenuses are given by an equation of the form $h(x)+g(y)=1$, there they satisfy the conditions $h(x), g(y)$ and are defined in each triangular element as follows: 


$$
\begin{array}{ll}
\mathrm{T}_{1}: h(x)=x^{2}, g(y)=y, & \mathrm{~T}_{2}: h(x)=-x, g(y)=y, \\
\mathrm{~T}_{3}: h(x)=x^{2}, g(y)=y^{2}, & \mathrm{~T}_{4}: h(x)=x^{2}, g(y)=y^{2} .
\end{array}
$$

Let a function $f(x, y)$ be defined in a certain area (Fig. 4 b):

$$
f(x, y)=\left\{\begin{array}{l}
0.5, \quad 0<x<1,0<y<1-x^{2}, \\
-x+1, \quad-1<x<0,0<y<1+x, \\
x^{2}+y^{2}, \quad-\sqrt{1-y^{2}}<x<\sqrt{1-y^{2}},-1<y<0 .
\end{array}\right.
$$

Therefore, the function $f(x, y)$ on the lines of triangulation has discontinuities of the first kind, but not on all lines. As initial data we will use the traces of a given function on the lines of triangular elements:

$$
\begin{aligned}
\mathrm{T}_{1}: & \varphi p(y)=f(+0, y)=0.5, \quad \psi p(x)=f(y,+0)=0.5, \\
& \eta m(x)=f\left(x, 1-x^{2}-0\right)=0.5 \Rightarrow \eta m(y)=0.5 \\
\mathrm{~T}_{2}: & \varphi m(y)=f(-0, y)=1, \quad \psi p(x)=f(x,+0)=-x+1, \\
& \eta p(y)=f(x, 1+x+0)=-x+1 \\
\mathrm{~T}_{3}: & \varphi m(y)=f(-0, y)=y^{2}, \quad \psi m(x)=f(x,-0)=x^{2}, \\
& \eta p(x)=f\left(x,-\sqrt{1-x^{2}}+0\right)=1 \\
\mathrm{~T}_{4}: & \varphi p(y)=f(+0, y)=y^{2}, \quad \psi m(x)=f(x,-0)=x^{2}, \\
& \eta m(x)=f\left(x,-\sqrt{1-x^{2}}-0\right)=1
\end{aligned}
$$

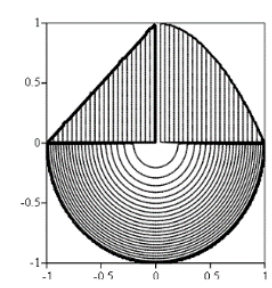

a)

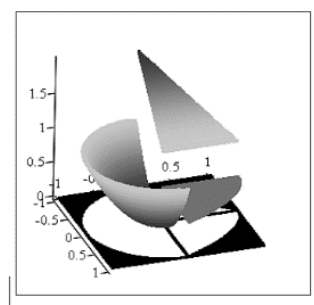

b)

Fig. 4. a) areas of the function definition; b) the form of the function $f(x, y)$

Let us construct the operator $L 1(x, y)$ on $\mathrm{T}_{1}$. By the theorem 5 it has a form

$$
\begin{gathered}
L 1 f(x, y)=L_{1} f(x, y)+L_{2}(x, y)-L_{12}(x, y) ; \\
L_{1} f(x, y)=h(x) \cdot \eta m(y)+g(y) \cdot \eta m(x)=x^{2} \cdot 0,5+y \cdot 0,5 \\
L_{2} f(x, y)=\psi p(x)+\varphi p(y)-\varphi p(0)=0,5+0,5-0,5=0,5 \\
L_{1} L_{2} f(x, y)=h(x)(\varphi p(y)+\mu p(y)-\varphi p(0))+g(y)(\eta m(0)+\mu m(x)-\psi p(x))
\end{gathered}
$$




$$
\begin{gathered}
=x^{2}(0,5+0,5-0,5)+y(0,5-0,5+0,5)=0,5 x^{2}+0,5 y \\
L 1 f(x, y)=0,5 x^{2}+0,5 y+0,5-0,5 x^{2}-0,5 y=0,5 .
\end{gathered}
$$

That is, the linear function of the interlination operator is restored exactly.

Operators L2,L3,L4 are constructed in a similar way. As a result, we obtain the discontinuous interlinaton operator, which completely coincides with the given function (Fig 4b).

\section{Construction of the Discontinuous Interlination Operator by Arbitrary Triangular Elements}

Let us consider a triangular element $\mathrm{T}_{i}, i=\overline{1, n}$ whose sides are defined by equations

$$
\begin{gathered}
\Gamma_{k}^{i}: \omega_{k}^{i}(x, y)=x \cdot \omega_{k 1}^{i}+y \cdot \omega_{k 2}^{i}-\gamma_{k}^{i}, \quad k=1,3, i=\overline{1, n},\left(\omega_{k 1}^{i}\right)^{2}+\left(\omega_{k 2}^{i}\right)^{2}=1, \\
\Delta_{123}^{i}=\left|\begin{array}{lll}
\omega_{12}^{i} & \omega_{11}^{i} & -\gamma_{1}^{i} \\
\omega_{22}^{i} & \omega_{21}^{i} & -\gamma_{2}^{i} \\
\omega_{32}^{i} & \omega_{31}^{i} & -\gamma_{3}^{i}
\end{array}\right| \neq 0, \Delta_{k \ell}^{i}=\left|\begin{array}{cc}
\omega_{k 1}^{i} & \omega_{k 2}^{i} \\
\omega_{\ell 1}^{i} & \omega_{\ell 2}^{i}
\end{array}\right| \neq 0, \quad k \neq \ell, \tau_{k}^{i}=\left(\omega_{k 2}^{i},-\omega_{k 1}^{i}\right), k=\overline{1,3} .
\end{gathered}
$$

Let $A_{k l}^{i}=\left(x_{k l}^{i}, y_{k l}^{i}\right)-$ is a solution of the systems of equation:

$\omega_{k}^{i}(x, y)=0, \omega_{\ell}^{i}(x, y)=0, k \neq \ell, k, \ell=\overline{1,3}$, which are the vertices of a given triangle.

We consider the given one-sided traces of the function $f(x, y)$ on the lines $\Gamma_{k}^{i}$ (below and above the line):

$$
\begin{gathered}
\varphi m_{k}^{i}(x, y)=f\left(x,\left(\gamma_{k}-x \omega_{k 1}\right) / \omega_{k 2}-0\right), \\
\varphi p_{k}^{i}(x, y)=f\left(x,\left(\gamma_{k}-x \omega_{k 1}\right) / \omega_{k 2}+0\right) \text { or } \\
\psi m_{k}^{i}(x, y)=f\left(\left(\gamma_{k}-y \omega_{k 2}\right) / \omega_{k 1}-0, y\right), \\
\psi p_{k}^{i}(x, y)=f\left(\left(\gamma_{k}-y \omega_{k 2}\right) / \omega_{k 1}+0, y\right) .
\end{gathered}
$$

Theorem 7. Let $f(x, y) \in C^{2}\left(\mathrm{~T}_{i}\right), i=\overline{1, n}$. If the traces of the functions $f(x, y)$ satisfy at the points $A_{k l}^{i}$ the Nikolsky condition [3], which can be written as

$$
\begin{gathered}
\varphi p_{3}^{i}\left(x_{13}^{i}, y\right)=\varphi m_{1}^{i}\left(x_{13}^{i}, y\right), \quad \varphi m_{1}^{i}\left(x_{12}^{i}, y\right)=\psi m_{2}^{i}\left(x, y_{12}^{i}\right), \\
\psi m_{2}^{i}\left(x, y_{32}^{i}\right)=\varphi p_{3}^{i}\left(x, y_{32}^{i}\right)
\end{gathered}
$$

then the operator 


$$
\begin{aligned}
& O^{i} f(x, y)=\frac{\omega_{1}^{i}(x, y)}{\omega_{1}^{i}\left(A_{23}^{i}\right)}\left(\psi m _ { 2 } ^ { i } \left(A_{23}^{i}-\frac{\tau_{2}^{i}}{\Delta_{23}^{i}} \omega_{3}^{i}(x, y)+\right.\right. \\
& \left.+\varphi p_{3}^{i}\left(A_{23}^{i}-\frac{\tau_{3}^{i}}{\Delta_{32}^{i}} \omega_{2}^{i}(x, y)\right)-\varphi p_{3}^{i}\left(A_{23}^{i}\right)\right)+\frac{\omega_{2}^{i}(x, y)}{\omega_{2}^{i}\left(A_{13}^{i}\right)}\left(\varphi m_{1}^{i}\left(A_{13}^{i}-\frac{\tau_{1}^{i}}{\Delta_{13}^{i}} \omega_{3}^{i}(x, y)\right)+\right. \\
& \left.+\varphi p_{3}^{i}\left(A_{13}^{i}-\frac{\tau_{3}^{i}}{\Delta_{31}^{i}} \omega_{1}^{i}(x, y)\right)-\varphi m_{1}^{i}\left(A_{13}^{i}\right)\right)+\frac{\omega_{3}^{i}(x, y)}{\omega_{3}^{i}\left(A_{12}^{i}\right)}\left(\varphi m_{1}^{i}\left(A_{12}^{i}-\frac{\tau_{1}^{i}}{\Delta_{12}^{i}} \omega_{2}^{i}(x, y)\right)+\right. \\
& \left.+\psi m_{2}^{i}\left(A_{12}^{i}-\frac{\tau_{2}^{i}}{\Delta_{21}^{i}} \omega_{1}^{i}(x, y)\right)-\psi m_{2}^{i}\left(A_{12}^{i}\right)\right) .
\end{aligned}
$$

is an interlination spline in $T_{i}$ which has properties

$$
\begin{aligned}
& \left.O^{i} f(x, y)\right|_{\Gamma_{1}: \omega_{1}^{i}(x, y-0)=0}=\left.\varphi m_{1}^{i}(x)\right|_{\Gamma_{1}: \omega_{1}^{i}(x, y-0)=0} ; \\
& \left.O^{i} f(x, y)\right|_{\Gamma_{2}: \omega_{2}^{i}(x, y-0)}=\left.\psi m_{2}^{i}(x)\right|_{\Gamma_{2}: \omega_{2}^{i}(x, y-0)=0} ; \\
& \left.O^{i} f(x, y)\right|_{\Gamma_{3}: \omega_{3}^{i}(x, y+0)=0}=\left.\varphi p_{3}^{i}(x)\right|_{\Gamma_{3}: \omega_{3}^{i}(x, y+0)=0}
\end{aligned}
$$

Theorem 8. If $f(x, y) \in C^{(2,2)}\left(\mathrm{T}^{i}\right), i=\overline{1, n}$, then the residual

$$
\begin{gathered}
R^{i} f(x, y)= \\
=\frac{\omega_{1}^{i}(x, y)}{\omega_{1}^{i}\left(A_{23}^{i}\right)} \int_{0}^{\omega_{2}^{i}(x, y)} \int_{0}^{\omega_{3}^{i}(x, y)} f^{(1,1)}\left(A_{23}^{i}-\frac{\tau_{2}^{i}}{\Delta_{23}^{i}} t_{3}-\frac{\tau_{3}^{i}}{\Delta_{32}^{i}} t_{2}\right) d t_{2} d t_{3}+ \\
+\frac{\omega_{2}^{i}(x, y)}{\omega_{2}^{i}\left(A_{13}^{i}\right)} \int_{0}^{\omega_{1}^{i}(x, y)} \int_{0}^{\omega_{3}^{i}(x, y)} f^{(1,1)}\left(A_{13}^{i}-\frac{\tau_{1}^{i}}{\Delta_{13}^{i}} t_{3}-\frac{\tau_{3}^{i}}{\Delta_{31}^{i}} t_{1}\right) d t_{1} d t_{3}+ \\
+\frac{\omega_{3}^{i}(x, y)}{\omega_{3}^{i}\left(A_{12}^{i}\right)} \int_{0}^{\omega_{1}^{i}(x, y)} \int_{0}^{\omega_{2}^{i}(x, y)} f^{(1,1)}\left(A_{12}^{i}-\frac{\tau_{1}^{i}}{\Delta_{12}^{i}} t_{2}-\frac{\tau_{2}^{i}}{\Delta_{21}^{i}} t_{1}\right) d t_{1} d t_{2} .
\end{gathered}
$$

Remark. If the one-sided traces on the same line coincide, then we get a continuous interlination spline.

Example 4. Let the area of the definition of a discontinuous function $f(x, y)$ be a triangle $\mathrm{T}$ (Fig. 5), whose sides are given by equations $\omega 1(x, y)=0, \omega 2(x, y)=0, \omega 3(x, y)=0$ :

$$
\begin{aligned}
& \omega 1(x, y)=x / \sqrt{50}+7 y / \sqrt{50}-3 / \sqrt{50}, \\
& \omega 2(x, y)=-4 x / \sqrt{41}+5 y / \sqrt{41}-1,2 / \sqrt{41}, \\
& \omega 3(x, y)=5 x / \sqrt{29}+2 y / \sqrt{29}-5,1 / \sqrt{29} .
\end{aligned}
$$

If we solve the pairwise systems of the above equations, we will get the points of intersection of the sides of the triangle: $A_{12}(0,2 ; 0,4), A_{23}(0,7 ; 0,8), A_{13}=(0,9 ; 0,3)$. 
And we define the function $f(x, y)$ as follows:

$$
f(x, y)= \begin{cases}x^{2}+y^{2}, & (x, y) \in \mathrm{T}, \\ 0, & (x, y) \notin \mathrm{T} .\end{cases}
$$

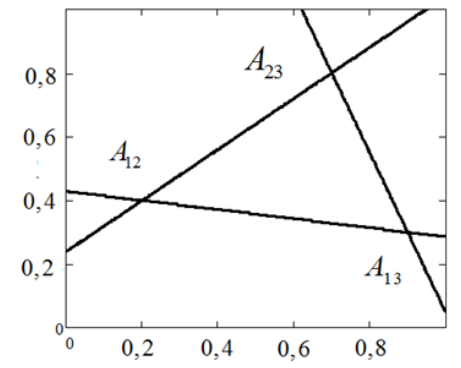

$a$

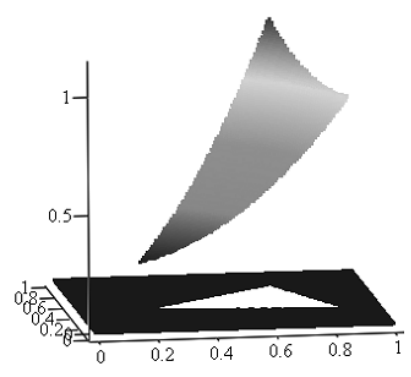

b

Fig. 5. Function $f(x, y): a$ - the area of definition; $b$ - the graphical representation

Therefore, the function has discontinuities on the lines of a given triangle and has the following traces on these lines (below and above the lines respectively):

$$
\begin{aligned}
& \varphi m_{1}(x, y)=0, \varphi p_{1}(x, y)=1,02 x^{2}-0,22 x+0,184, \\
& \psi m_{2}(x, y)=0, \varphi p_{2}(x, y)=2,56 y^{2}-0,75 y+0,09, \\
& \varphi m_{3}(x, y)=7,25 x^{2}-12,75 x+6,5025, \varphi p_{3}(x, y)=0 .
\end{aligned}
$$

Let us check the fulfilment of Nikolsky's conditions:

$$
\begin{aligned}
& \left.\varphi p_{1}(x, y)\right|_{\omega 2(x, y-0)=0}=\left.\psi m_{2}(x, y)\right|_{\omega 1(x-0, y)=0}=0,2, \\
& \left.\psi m_{2}(x, y)\right|_{\omega 3(x-0, y)=0}=\left.\varphi p_{3}(x, y)\right|_{\omega 2(x, y-0)=0}=1,13, \\
& \left.\varphi p_{2}(x, y)\right|_{\omega 3(x+0, y)=0}=\left.\varphi m_{3}(x, y)\right|_{\omega 1(x-0, y)=0}=0,9 .
\end{aligned}
$$

Thus, the conditions of Theorem 7 are satisfied. Then we construct an interlination spline according to the formula (4), the graph of which is shown in Fig. 6(b). Fig. 6(a) shows a discontinuous interpolation spline approximating for comparison the same discontinuous function. 


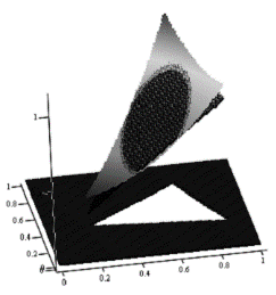

a)

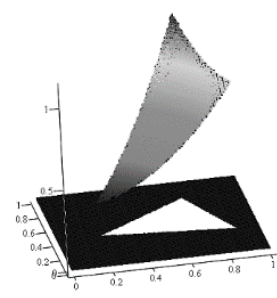

b)

Fig. 6. A graphic representation of the given discontinuous function $f(x, y)$ and discontinuous splines: $a$ ) interpolation; $b$ ) interlination

\section{Conclusions}

The paper presents a method for approximating a discontinuous function of two variables by its known traces on a system of arbitrary lines. For this purpose, a discontinuous interlination operator was constructed using triangular elements. It was shown that the proposed interlination operator approximates the discontinuous function more precisely than the classical interpolation operator. In the future works, based on the proposed operator, authors will be able to construct a method for approximating a discontinuous function using a partition of the domain into triangular elements.

\section{References}

Ageev, A.L, and Antonova, T.V., Algorithms of increased precision of approximation of discontinuity lines of noisy function. Trudy Inst. Mat. i Mekh. UrO RAN, vol. 23, 2, pp. 1021. (2017).

Ageev, A.L., and. Antonova, T.V., On the localization of discontinuities of the first kind for a function of bounded variation. Proceedings of the Steklov Institute of Mathematics, vol. 280, pp. 13-250. (2013).

Beckermann, B., Reduction of the Gibbs phenomenon for smooth functions with jumps by the $\varepsilon$ algorithm. Journal of Computational and Applied Mathematics, vol. 219, 2, pp. 329-349. (2008).

Bozzini, M., and Rossini, M., The detection and recovery of discontinuous curves from scattered data. Journal of Computational and Applied Mathematics, 240, pp. 148-162. (2013).

Derevtsov, E.Y, Maltseva, S.V., Svetov, I.E. Determination of Discontinuities of a Function in a Domain with Refraction from Its Attenuated Ray Transform. J. Appl. Ind. Math., 12, pp. 619-641. (2018).

Faridani, A., Finch, D. V., Ritman, E. L., Smith, K. T., Local tomography. II. SIAM J. Appl. Math., 57 (4), pp. 1095-1127. (1997).

Gorban, A.N., Approximation of continuous functions of several variables by an arbitrary nonlinear continuous function of one variable, linear functions, and their superposition. Appl. Math. Lett., vol. 11, 3, pp. 45-49. (1998)

Lombardini, R., Acevedo, R., Kuczala, A., Keys, K.P., and Goodrich, C.P., Higher-order wavelet reconstruction/differentiation filters and Gibbs phenomena. Journal of Computational Physics, 15, pp. 244-262. (2016). 
Louis, A. K., Feature Reconstruction in Inverse Problems. Inverse Problems, 27 (6), Art. 065010. (2011).

Lytvyn, O.M., Pershina, Y.I., Litvin, O.O., Kulyk, S.I, and Shumeyko N.A., New method of restoration of internal structure 3D bodies by means of projections which arrive from a computer tomography. 6th World Congress in Industrial Process Tomography, pp. 429-436 (2010).

Lytvyn, Oleg N., Pershina, Yulia I., Reconstruction of 3 - D objects with use interflation of functions. Signal and image processing. Proceeding of the Second IASTED International Multi-Conference on Automation, Control, and Information Technology, pp. 274-279 (2005).

Lytvyn, O.M., Pershyna, I.I., Lytvyn, O.O., and Kulyk, S.I., Mathematical modelling of discontinuous processes in a computer tomography by means of discontinuous splines. 7 th World Congress in Industrial Process Tomography, pp.441-450 (2013).

Lytvyn, O.N., and Pershina, Yu.I., Approximation of discontinuous function of two variables by approximating discontinuous bilinear spline using the least-squares method (rectangular elements). Journal of Automation and Information Sciences, vol. 44, 5, pp. 48-56 (2012).

Lytvyn, O.M., Pershyna, I., Nechuiviter, O, Lytvyn, O.O., Restoration of Discontinuous Functions by Interpolation Data Using Rectangular Elements. 9th International Conference on Advanced Computer Information Technologies, pp. 40-43. (2019)

Mezhuyev, V., Lytvyn, Oleg M., Pershyna, I., Nechuiviter O., Lytvyn Oleg O., Algorithm for the Reconstruction of the Discontinuous Structure of a Body by Its Projections along Mutually Perpendicular Lines. Proceedings of 2018 7th International Conference on Software and Computer Applications, pp. 158-163. (2018)

Milovanovic, G.V.and Rassias, M.Th., Analytic number theory. Approximation theory and special functions. Springer, New York (2014).

Radon, J., Über die Bestimmung von Functionen durch ihre Integralverte Längs gewisser Manningfaltigkeiten. Ber. Verh. Sächs. Acad. Wiss. Leipzig Math. Nat. Kl, vol. 69, pp. 262277. (1917).

Ramachandran, G.N., and Lakshminarayanan, A.V., Three-dimensional reconstruction from radiograph and electron micrographs: application of convolutions instead of Fourier transforms. Proc. Nat. Acad. Sci. US, 68, pp. 2236-2240. (1997).

Rossini, M. Detecting discontinuities in two-dimensional signals sampled on a grid. Journal of Numerical Analysis, Industrial and Apply Mathematics, vol. 1, pp. 1-13. (2007).

Suresh, V., Koteswarao Rao, S., Thiagarajan G., and Das R.P., Denoising and detecting discontinuities using wavelets. Indian Journal of Science and Technology, 9(19), pp. 1-4. (2016).

Tampos, A.L., Lope, J.E.C, and Hesthaven, J.S., Accurate reconstruction of discontinuous functions using the singular pade-Chebyshev method. International Journal of Applied Mathematics, vol. 42, 4, pp. 242-249. (2013). 